\title{
Efeitos do método Pilates em diástase abdominal após período gestacional
}

\author{
Effects of Pilates method on abdominal diastasis after pregnancy \\ Efectos del método Pilates em la diástasis abdominal después del embarazo
}

Recebido: 02/09/2021 | Revisado: 08/09/2021 | Aceito: 09/09/2021 | Publicado: 12/09/2021

\author{
Kaellen Rodrigues de Castro \\ ORCID: https://orcid.org/0000-0001-5222-9382 \\ Instituto Educacional Santa Catarina, Brasil \\ Faculdade Guaraí, Brasil \\ E-mail: kaellenrodrigues@gmail.com \\ Wênia Caetano de Oliveira \\ ORCID: https://orcid.org/0000-0002-1246-975X \\ Instituto Educacional Santa Catarina, Brasil \\ Faculdade Guaraí, Brasil \\ E-mail: weniacartano20@gmail.com \\ Lécia Kristine Lourenço \\ ORCID: https://orcid.org/0000-0002-0531-7221 \\ Instituto Educacional Santa Catarina, Brasil \\ Faculdade Guaraí, Brasil \\ E-mail: leciakristine@yahoo.com.br
}

\begin{abstract}
Resumo
A diástase abdominal fisiológica, acomete a maioria das mulheres durante o período gestacional devido ao aumento da circunferência do abdômen, necessário para que ocorra o desenvolvimento do feto, contudo, caso a separação dos retos abdominais se torne exacerbada, a diástase abdominal passa a classificar-se como patológica e pode resultar em agravos à saúde e à autoestima da mulher. Portanto, o objetivo deste artigo é apresentar o método Pilates como tratamento na diástase abdominal após período gestacional, por meio de pesquisas realizadas nas plataformas do Google Acadêmico, PubMed e Scientific Electronic Library Online (Scielo), em busca de artigos científicos publicados entre os anos de 2015 e 2021. Mediante às pesquisas executadas, obtém-se que o método Pilates através de seus princípios e técnicas, com ênfase na contração da musculatura nomeada power house, é capaz de reduzir a distância entre os feixes musculares dos músculos retos abdominais, podendo assim, ser um importante recurso no tratamento da diástase abdominal após o período gestacional.
\end{abstract}

Palavras-chave: Pilates; Gestação; Diástase abdominal; Benefícios do pilates; Gravidez.

\begin{abstract}
Physiological abdominal diastasis affects most women during the gestational period due to the increased circumference of the abdomen, necessary for the development of the fetus to occur, however, if the separation of the rectus abdominis becomes exacerbated, abdominal diastasis begins to classify if pathological and may result in damage to women's health and self-esteem. Therefore, the aim of this article is to present the Pilates method as a treatment for abdominal diastasis after pregnancy, through research carried out on the Academic Google, PubMed and Scientific Electronic Library Online (Scielo) platforms, in search of scientific articles published between the years of 2015 and 2021. Through the research carried out, it is possible to obtain that the Pilates method through its principles and techniques, with emphasis on contraction of muscles named power house, it's able to reduce the distance between rectus abdominis muscles bundles muscles', which may thus, be an important resource in the treatment of abdominal diastasis after the gestational period.
\end{abstract}

Keywords: Pilates; Gestation; Abdominal diastasis; Pilates' benefits; Pregnancy.

\section{Resumen}

La diástasis abdominal fisiológica afecta a la mayoría de las mujeres durante el período gestacional debido al aumento de la circunferencia del abdomen, necesario para que ocurra el desarrollo del feto, sin embargo, si la separación del recto abdominal se agrava, la diástasis abdominal se clasifica como patológica y puede resultar en daño a la salud y la autoestima de las mujeres. Por tanto, el objetivo de este artículo es presentar el método Pilates como tratamiento para la diástasis abdominal post-embarazo, a través de una investigación realizada en las plataformas Academic Google, PubMed y Scientific Electronic Library Online (Scielo), en busca de artículos científicos publicados entre 2015 y 2021. Mediante las investigaciones realizadas, es posible lograr que el método Pilates a través de sus principios y técnicas, con énfasis en la contracción de los músculos llamados power house, sea capaz de reducir la distancia entre los haces musculares del recto abdominal, podendo así, ser un recurso importante para el tratamiento de la diástasis abdominal después del período gestacional.

Palabras clave: Pilates; Gestación; Diástasis abdominal; Beneficios de pilates; Embarazo. 


\section{Introdução}

No período gestacional a mulher enfrenta diversas modificações de cunho físico ou morfológico, trazendo complicações ao nível biomecânico, atingindo sua estrutura muscular havendo um déficit de força e consequentemente alterações posturais (Bráz, Silva, \& Silva, 2018) Entretanto, o puerpério é uma etapa de transição no qual a mulher passa pela fase da gestação e entra no estado pré-gravídico. Esse é um período no qual o corpo está retornando a sua estrutura anatômica, com modificações geradas através de fatores hormonais, físicos e biomecânicos. Dentre esses fatores, podemos destacar a diástase abdominal, uma alteração que atinge grande maioria das mulheres, permanecendo ao longo da vida, caso não seja tratada de forma a reverter esse quadro (Urbano, Barbosa, Junior, Martelli, \& Delbim, 2019).

Tal alteração se dá pelo estiramento ou desunião dos músculos do reto abdominal no intuito de possibilitar o crescimento do feto no ventre da mulher (Fei et al., 2021; Kroetz, \& Santos, 2015; Nahabedian, 2018). Esses músculos são responsáveis pela sustentação do tronco, proteção visceral e sustentação postural além de estar relacionado a respiração (Urbano et al., 2019). Em relação aos sintomas, é indolor e não apresenta desconforto, porém pode causar lombalgias devido a alteração do músculo reto abdominal, implicando no power house e nas gestações futuras (Leite, \& Araújo, 2012).

A diástase abdominal é classificada como patológica, quando há um distanciamento dos feixes musculares maior que 3cm (Leite, \& Araújo, 2012). Em contraposição segundo Leite \& Araújo (2012) e Müller \& Silva (2018) relatam que ainda não há evidência científica atual que pontue o valor numérico exato para considerar uma diástase em patologia. Porém pode trazer prejuízo no quesito biomecânico, além de influenciar consideravelmente na depreciação da autoestima da mulher (Jessen, Öberg, \& Rosenberg, 2019).

Um método bastante eficaz usufruído nesses casos de diástase abdominal é o método Pilates, fundado por Joseph Humbertus Pilates (1880-1967). Nascido na Alemanha, enfrentava problemas de saúde que influenciava diretamente à sua estrutura física, já que ele era raquítico. Essa técnica de reabilitação relacionada a prática de atividade física, objetiva equilíbrio, flexibilidade e fortalecimento, obedecendo a fisiologia de todo indivíduo e promovendo inúmeros benefícios (Kroetz, \& Santos, 2015).

Contudo, a mulher que apresentar a diástase abdominal deve ter um plano de tratamento individualizado onde o método entrará como recurso a tratar de maneira a auxiliar no acometimento muscular devido a gestação ou após o parto, visto que evidências comprovam que a diástase pode ser causada também durante o trabalho de parto devido ao esforço físico necessário para expulsão do feto (Pereira, Rego, Pinto, \& Blanco, 2020).

Segundo Fernandes \& Santos (2016), os exercícios executados devem ser realizados com a tonificação dos músculos da região do abdômen, nomeado "power house" ou centro de força, concebido pelas quatro camadas do abdômen (reto abdominal, oblíquo externo, oblíquo interno e transverso do abdome), assoalho pélvico e eretores profundos da coluna, tornando-se tais musculaturas encarregadas pela estabilidade estática e dinâmica do corpo.

Vale ressaltar que o método Pilates é baseado em seis princípios, sendo eles: respiração, concentração, centralização, precisão, fluidez e controle (Kroetz, \& Santos, 2015). Dentre as vantagens encontram-se a ativação da circulação sanguínea, aperfeiçoamento do condicionamento físico, promoção de elasticidade, aptidão muscular e o melhor alinhamento muscular, retomando a autoestima e bem-estar (Bom-fim, Martins, \& Fernandes, 2020).

Relatos da literatura comprovam que, quanto mais prévia ocorrer a intervenção, mais acelerado será o retorno da musculatura do reto abdominal, ocasionada pela alteração anatômica e fisiológica durante o período gestacional (Urbano et al., 2019).

Portanto, essa revisão literária propõe-se a identificar e analisar o efeito do método Pilates após o período gestacional, por meio de técnicas para a redução da diástase do músculo reto abdominal e a medida dinâmica da distância inter-retos durante os exercícios abdominais. 


\section{Metodologia}

Concerne a uma revisão literária, de cunho qualitativo, com base em fundamentações científicas, a partir de fontes secundárias que buscam evidenciar os benefícios do método Pilates em diástase abdominal após período gestacional (Pereira, Shitsuka, Parreira, \& Shitsuka, 2018).

Para a presente revisão foi realizado um levantamento de dados nas bases de dados Google Acadêmico, Scientific Electronic Library Online (Scielo) e PubMed de artigos publicados entre os anos de 2015 à 2021, salvo os de maior relevância antes do período determinado, em língua portuguesa e inglesa, além de incorporação de trabalhos de conclusão de curso publicados em repositórios, utilizando como descritores isolados ou em combinação: Pilates, gestação, diástase abdominal, benefícios do Pilates, gravidez, combinados entre si.

Após análise seletiva e criteriosa dos dados coletados, foram excluídos da revisão artigos infundamentados e/ou que não obedeciam às especificações buscadas. Para uma maior confiança na seleção dos artigos, a avaliação dos resumos e do texto completo foi realizada por dois pesquisadores, de forma autônoma, seguindo rigorosamente os critérios de inclusão e exclusão. Nos casos em que houve divergência entre os pesquisadores, um terceiro revisor foi consultado.

\section{Resultados e Discussão}

\subsection{Alterações Gestacionais}

O período gestacional está diretamente relacionado aos ajustes fisiológicos, psicoemocionais e sociais, ocorridos no organismo materno que se prepara para o desenvolvimento do bebê (Vasconcelos, Oliveira, Alves, Santos, Silva, \& Lira, 2017). É caracterizado por uma série de eventos que se iniciam com a fertilização e encerram-se com o parto (Pereira et al., 2020). Com duração de aproximadamente 40 semanas, a forma e a necessidade do corpo mudam com constância, conforme a gestação avança (Kroetz, \& Santos, 2015; Pereira et al., 2020).

Segundo Kroetz e Santos (2015), em nenhum outro período da vida, ocorrem tantas mudanças na fisiologia do corpo humano em um curto espaço de tempo, como na gestação. Por tratar-se de verdadeiras transformações e adequações, afeta todos os sistemas, como o endócrino, o cardiovascular, o hematopoiético, o tegumentar, o digestivo, o urinário, o articular, e o de maior interesse para o presente estudo, o musculoesquelético (Pereira et al., 2020).

As alterações fisiológicas decorrentes desse período, acontecem devido a ação hormonal do estrogênio, da progesterona e da relaxina, principalmente (Fei et al., 2021; Urbano et al., 2019). Dentre essas alterações podemos citar o aumento das glândulas mamárias, da frequência e consequentemente do débito cardíaco, déficit respiratório, enjoos e dificuldade na digestão, urgência miccional, entre outras (Pereira et al., 2020).

Uma das modificações biomecânicas mais evidentes, pode ser observada na parede abdominal, em decorrência da expansão do útero com o crescimento do bebê (Figura 1). Conforme o útero expande, há um estiramento na musculatura abdominal, predominantemente sobre o músculo reto abdominal, alongado em até $20 \mathrm{~cm}$ a mais que seu tamanho natural. O afastamento desse músculo é denominado como diástase do músculo reto abdominal (DMRA), e pode acontecer não somente durante a gravidez, mas também no período expulsivo, imediatamente após o parto ou nas primeiras semanas do puerpério (Fei et al., 2021; Urbano et al., 2019). 
Figura 1 - Estiramento da musculatura abdominal.
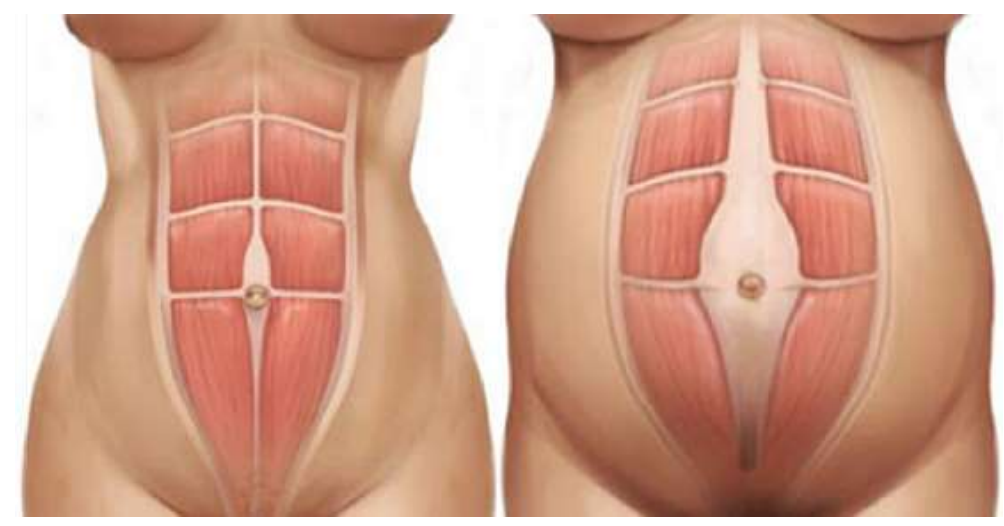

Fonte: https://fisiostudiopilates.com.br/diastase-do-abdome-tudo-o-que-voce-precisa-saber/

Fei et al. (2021) e Urbano et al. (2019) afirmam em seus respectivos estudos que, fatores como obesidade, multiparidade, polidrâmnio e flacidez da musculatura abdominal pré-gravídica, estão relacionados a um maior estiramento do reto abdominal, causando assim, uma DRMA mais acentuada.

\subsection{Diástase Abdominal}

O abdômen localiza-se na região central do corpo, tendo sua musculatura constituída pela junção das aponeuroses dos músculos reto abdominal, transverso do abdômen, oblíquo externo e oblíquo interno. Tais musculaturas são necessárias para diminuição do esforço de retração sobre a coluna vertebral, além do importante papel de integração ao core, desempenhando a função de sustentação e estabilização do tronco, manutenção da postura e mobilidade corporal (Fei et al., 2021; Lynders, 2019; Nahabedian, 2018; Urbano et al., 2019).

Podemos destacar o musculo reto abdominal como um dos principais músculos pelo fato de que proporciona ao corpo um aporte mecânico tanto dinâmico, quanto estático, pois contribui de forma íntegra na execução de movimentos como flexão lateral da coluna vertebral e flexão de tronco, além de ter influência na respiração profunda e proteção das vísceras (Urbano et al., 2019).

Fei et al. (2021) e Kroetz e Santos (2015) alegam que a diástase é acarretada pelo afastamento dessas musculaturas afim de conceder à ampliação do útero para o crescimento do feto, devido alterações durante o período gestacional, acometendo aproximadamente dois terços das gestantes podendo também ocorrer durante o período expulsivo, em seguida do parto, ou, na primeira semana após (Figura 2). Já segundo Müller e Silva (2018) a diástase do reto abdominal acomete todas as mulheres gestantes com prevalência de 52,4\% da sexta a oitava semana de pós-parto e permanece em 39,3\% aos seis meses de pós-parto e em $32,6 \%$ com doze meses após o parto. 


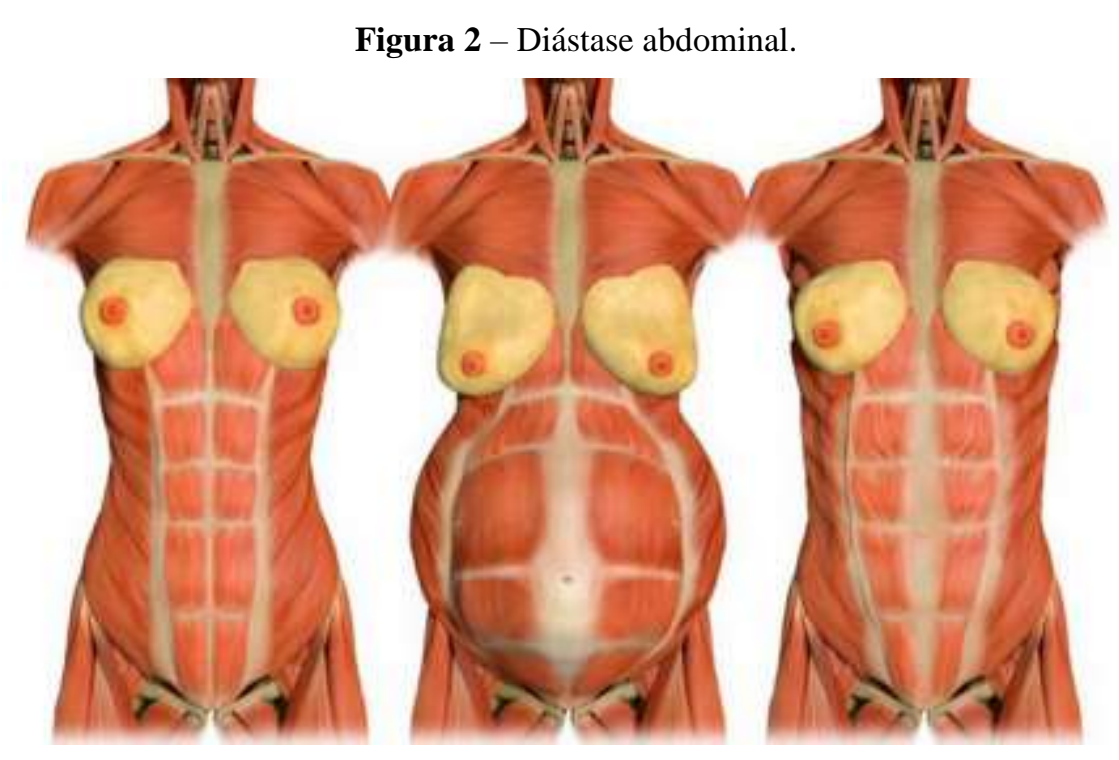

Fonte: https://www.hsnstore.pt/blog/saude-e-beleza/lesoes/diastase-abdominal/

De acordo com Leite e Araújo (2012), após uma pesquisa de campo envolvendo 100 puérperas assistidas nas maternidades Frei Damião e Cândida Vargas (município de João pessoa, Paraíba) obteve uma maior incidência da diástase supra umbilical associada à separação ao nível umbilical, podendo esta ter uma relação intimamente ligada ao fato de possuir mais de um ventre muscular. Porém, há pesquisas que evidenciam o acometimento em outras regiões como infra umbilical ou umbilical.

Outro fator relevante pelo qual a diástase abdominal é manifestada, é em relação ao tipo de parto, no qual os resultados da pesquisa anteriormente citada, constataram que mulheres que se sujeitaram ao parto normal foram as mais acometidas pela DMRA e isso pode ter relação com o trabalho de parto, devido a força intra-abdominal durante a expulsão do feto. No entanto, faz-se necessário mais estudos que envolvam a inclusão da DMRA em partos cesariana (Leite, \& Araújo, 2012).

Em relação às alterações musculoesqueléticas, pode provocar algumas complicações ao corpo da mulher, tanto de cunho físico como emocional (Kroetz, \& Santos, 2015). No quesito físico, tais alterações influenciam diretamente nas funções musculoesqueléticas, atingindo o padrão de estabilidade corporal, tendo uma predisposição a dor lombar e disfunções uroginecológicas (Fei et al., 2021; Jessen et al., 2019; Urbano et al., 2019). Já no âmbito emocional atinge de forma negativa questões estéticas, de autoimagem, pois na maioria dos casos o abdômen adquire um aspecto globoso (Fei et al., 2021; Kroetz, \& Santos, 2015; Pereira et al., 2020).

A avaliação da DMRA deve ser realizada por um profissional habilitado para obtenção de dados efetivos para assim dar início ao tratamento. Há duas formas de avaliação, sendo a palpação com os dedos em região de abdômen mais especificamente na linha alba (linha de cor branca, robusta localizada entre os retos abdominais), demonstrada na figura 3, na qual fará a mensuração de forma a identificar a largura e o comprimento da diástase (Vasconcelos et al., 2017). 
Figura 3 - Avaliação da diástase abdominal.

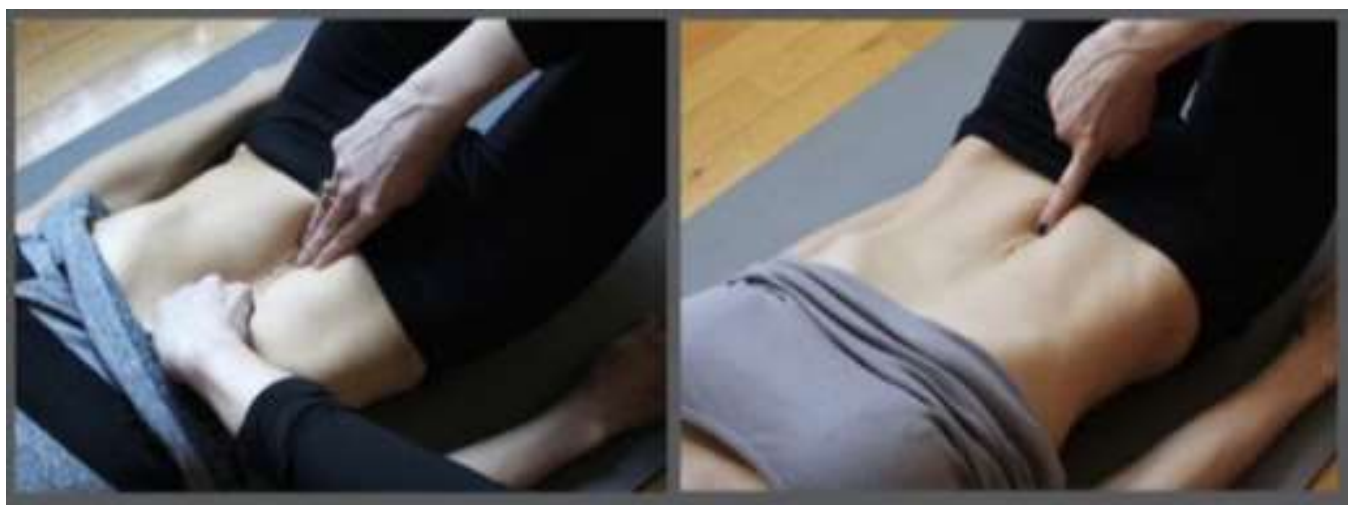

Fonte: https://fisiostudiopilates.com.br/diastase-do-abdome-tudo-o-que-voce-precisa-saber/

Outra forma de avaliar, é com o auxílio de um paquímetro que efetuará a estimação de maneira acurada (Figura 4). Para isso, é necessário solicitar que a puérpera se deite em decúbito dorsal, com os membros inferiores fletidos e pés fixados sobre o local que está deitada, em seguida, solicite a mesma que execute uma flexão de tronco (levando o queixo até os seios) para que o terapeuta possa utilizar o paquímetro e obter o distanciamento através da medida de centímetros, de maneira mais autêntica (Vasconcelos et al., 2017).

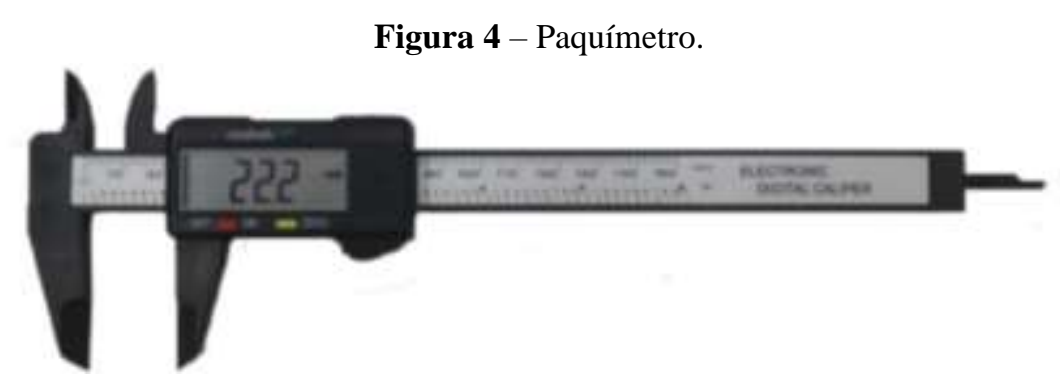

Fonte: https://profono.com.br/loja/paquimetro-digital-pro-fono/

Há três modos a reverter o quadro de diástase do reto abdominal, sendo assim, espontâneo no qual o próprio corpo retornará sua estrutura anatômica normal, a conservadora, podendo destacar o método Pilates pois, vai intervir de maneira a abranger todos os sistemas que facilitará a diminuição do afastamento de tal estrutura, e o tratamento cirúrgico, como, por exemplo, os procedimentos mais comuns: abdominoplastia e plicatura laparoscópica (Jessen et al., 2019; Mommers et al., 2017; Müller, \& Silva, 2018). Todavia, quando o corpo for incapaz de reverter esse quadro espontaneamente, o método mais recomendado nesse quesito é o conservador, haja vista que o tratamento cirúrgico pode trazer consigo complicações mais graves por se tratar de um procedimento extremamente invasivo (Müller, \& Silva, 2018).

\subsection{Método Pilates}

O método Pilates, originalmente chamado de Contrologia, foi criado no ano de 1920 pelo alemão Joseph Humbertus Pilates (Figura 5), que buscava lidar com as patologias que marcaram sua infância: asma, raquitismo e febre reumática. Baseado em suas experiências com práticas artísticas, esportivas e terapêuticas, Pilates criou um programa de exercícios com enfoque na coordenação da mente, do espírito e do corpo, de forma a trabalhar com os músculos do corpo e não "sobre" eles (Kroetz, \& Santos, 2015; Macedo, Oliveira, Gonçalves, \& Mascarenhas, 2018; Pereira et al., 2020). 
Figura 5 - Joseph Pilates realizando o movimento roll-over de seu método.

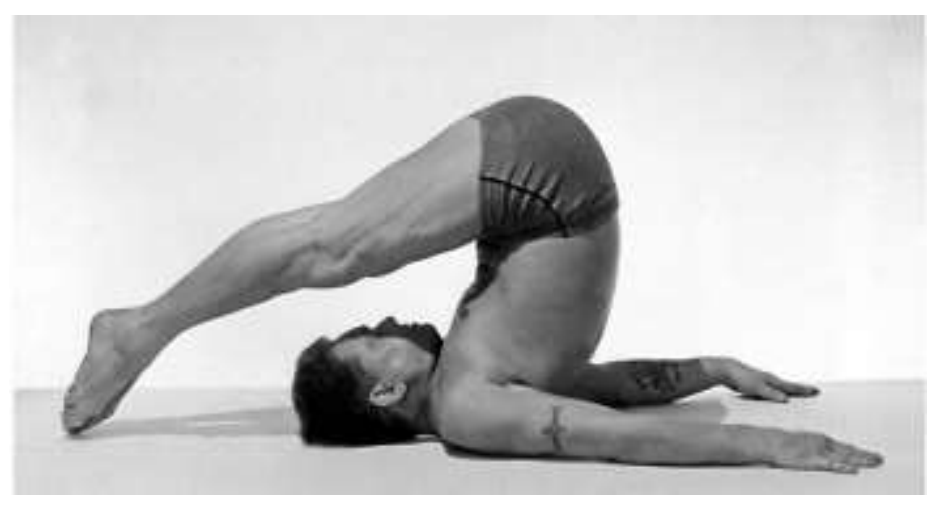

Fonte: https://www.pilatescenterbh.com.br/pilates/

Em 1967, decorrente de um incêndio em seu estúdio, Joseph Pilates inalou grande quantidade de fumaça tentando recuperar seus equipamentos, levando-o a morte de maneira trágica. Desde então, a Contrologia passou a receber o nome de Método Pilates, em homenagem a seu criador (Pereira et al., 2020). Trata-se de um método completo, que através da valorização da interação entre o corpo e a mente, busca desenvolver no praticante uma maior consciência corporal e uma postura mais equilibrada. Por meio da integração mente e corpo, pode-se observar um aperfeiçoamento na precisão do controle muscular, da força e da flexibilidade, qualificando os movimentos corporais (Bom-fim et al., 2020). Para que isso seja possível, o Pilates é estruturado em alguns princípios básicos: concentração, precisão, controle, respiração, centralização e fluidez (Bom-fim et al., 2020; Nascimento, \& Mejia, n.d.; Pereira et al., 2020).

Para a realização adequada de um movimento, faz-se necessário concentrar-se nos músculos e nas partes do corpo exigidos durante a execução do exercício, fazendo com que o sistema nervoso central (SNC) envie uma maior quantidade de impulsos nervosos para a realização da atividade, e assim, haja uma maior eficiência, surgindo daí o princípio da concentração (Pereira et al., 2020).

O princípio da precisão/controle, preconiza a técnica no momento da execução e não por meio da quantidade de repetições, dessa forma, a mente deve controlar os movimentos para que o alinhamento corporal seja mantido e não haja compensações, retarde a fadiga muscular e previna lesões advindas da instabilidade, recrutando apenas os músculos necessários (Pereira et al., 2020).

A respiração, um dos princípios mais importantes do Pilates, deve ser ensinada desde o primeiro contato com o método. Consiste na prática correta da inspiração, no preparo para iniciar o exercício e da expiração, durante sua execução, de forma a proporcionar uma apropriada oxigenação do sangue, a otimização da circulação tecidual, o relaxamento da musculatura inspiratória e a eliminação de gases nocivos, potencializando os benefícios obtidos com o exercício (Kroetz, \& Santos, 2015; Pereira et al., 2020).

Joseph Pilates, determinou como centro de força, em inglês Power House, os músculos abdominais, paraverterbrais lombares e assoalho pélvico, responsáveis por gerar o início da energia irradiada pelas extremidades. O último princípio aqui mencionado, refere-se à realização dos exercícios de forma harmoniosa, numa velocidade moderada, evitando movimentos em bloco e trabalhando simultaneamente diferentes grupos musculares. A fluidez dos movimentos é essencial para a redução da tensão e da rigidez, tornando-se primordial para a obtenção da concentração, precisão e controle dos movimentos (Pereira et al., 2020).

Os princípios do Pilates, abordados acima, permitem que o praticante obtenha um resultado máximo em todos os exercícios ao praticá-los corretamente. A relação entre os benefícios do método no tratamento da DMRA, se faz presente de 
forma indireta ainda nos princípios, haja vista que, é indispensável para a sua prática a contração da musculatura abdominal (Kroetz, \& Santos, 2015).

\subsection{Pilates na Diástase Abdominal}

A diástase do reto abdominal é frequentemente acometida em gestantes devido as mudanças hormonais causadas pela progesterona, estrogênio e relaxina à musculatura esquelética devido ao estiramento dos músculos do abdômen, necessário para o desenvolvimento do feto. Entretanto, logo após o período gestacional o corpo retorna ao estado pré-gravídico (Luna, Cavalcanti, Guendler, Brito, \& Oliveira, 2012).

A DMRA gera uma falha na integralidade da parede abdominal, falha essa que pode causar o aumento da gravidade da diástase, durante a realização de atividades que evidenciem a elevação da pressão intracavitária, portanto, é imprescindível o cuidado com tosses intensas e com a contração abdominal ao erguer objetos pesados (Bom-fim et al., 2020).

Estudos como o de Urbano et al. (2019), apontam o método Pilates como uma das terapias eficazes no tratamento da DMRA por proporcionar o fortalecimento da musculatura alterada, auxiliando no seu retorno ao estágio pré-gestação e prevenindo contra danos adicionais. É válido ressaltar que, o método em questão vem passando por várias adaptações conforme as compreensões do corpo humano e dos novos estilos de movimento, sendo assim, um método que supre as carências e individualidades de cada paciente (Silva et al., 2019).

O Pilates na diástase, especificamente no pós-parto consiste na recuperação da musculatura do reto abdominal em virtude da gestação, além de amplificar a aptidão dos movimentos já que o método busca a interação do corpo e da mente (Kroetz, \& Santos, 2015). Nesse caso, os exercícios fundamentam-se no fortalecimento dos músculos retais do abdômen e consequentemente aos músculos paravertebrais (Figura 6) (Urbano et al., 2019). Por isso a importância do Pilates na diástase, por se tratar de condicionamento muscular especificamente da região abdominal sem que haja compressão exagerada da musculatura (Pereira et al., 2020).

Figura 6 - Fortalecimento da musculatura abdominal.

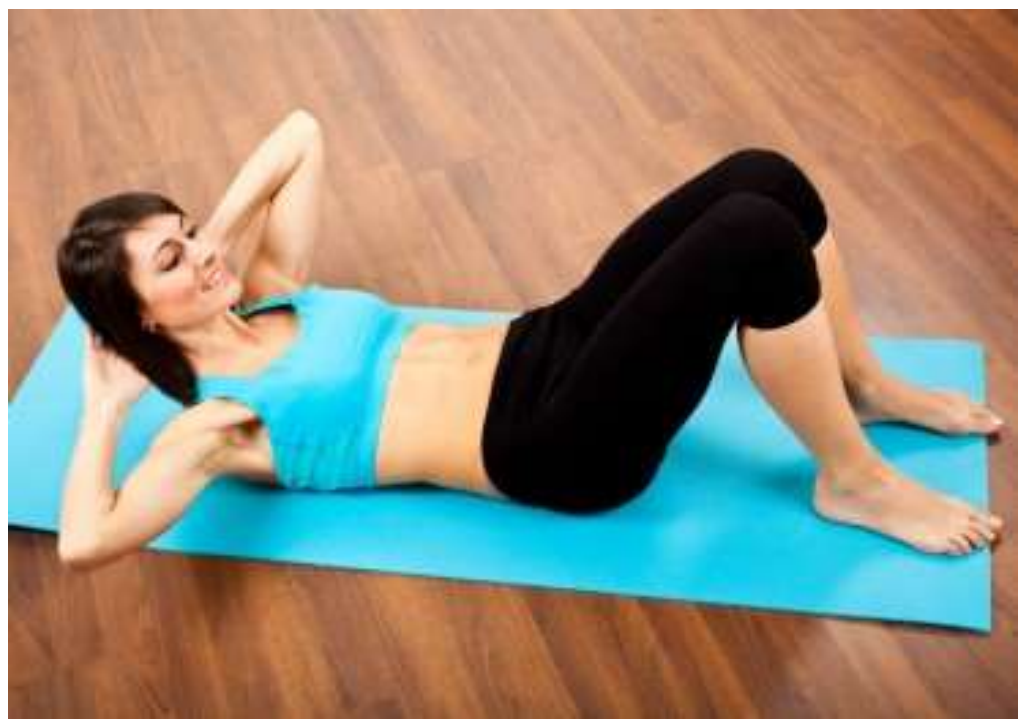

Fonte: http://cursostaopilatesjoinville.blogspot.com/2016/06/o-que-e-diastase-abdominal.html

A prática do Pilates na reabilitação da diástase pode ser realizada de duas maneiras, sendo elas: Pilates clássico, exercícios que utilizam de aparelhos como, Ladder Barrel, cadeira StepChair, Cadillac reformer; e Mat pilates (exercícios em 
solo) classificado pela utilização do peso corpóreo e força da gravidade na execução dos movimentos como resistência. Geralmente, o Mat Pilates servirá de base para execução dos exercícios com os aparelhos (Figura 6) (Luna et al., 2012).

Por ser um método aplicado em consonância com as necessidades individuais do praticante e baseado no recrutamento da musculatura profunda (power house) com enfoque na respiração, consequentemente age nos retos abdominais que pertencem ao grupo de músculos que exercem a sustentação do corpo e estão relacionados a estabilização pélvica, corrigindo assim, sua disfunção e promovendo o fortalecimento necessário para correção da DMRA (Pereira et al., 2020).

\section{Considerações Finais}

Diante da pesquisa, podemos concluir que a diástase pode ser considerada patológica quando há um afastamento exacerbado da musculatura do reto abdominal e por essa virtude, essa musculatura poderá não ser restituída a sua estrutura anterior espontaneamente, nesse caso, se não tratada pode gerar complicações à saúde por compensar em outras estruturas do corpo devido a perda do suporte muscular. Para reverter esse quadro, o método Pilates traz consigo inúmeros benefícios, ao se referir à um tratamento seguro, não invasivo e adaptativo, ou seja, a execução varia da necessidade de cada corpo, levando em consideração a avaliação antes da prática dos exercícios.

Apesar de todos os benefícios abordados, exaltando primordialmente o fortalecimento do abdômen, é um assunto ainda pouco discutido entre as mulheres, possivelmente por ser uma abordagem consideravelmente recente, à vista disso, torna-se um fator problemático, quando se trata da busca por tratamento. Por conseguinte, vale ressaltar a escassez de trabalhos científicos relacionados ao Pilates como tratamento isolado da diástase abdominal. Em virtude disso e da importância expressiva quando associada à saúde física e mental da mulher, faz-se necessário a produção de mais artigos científicos aprofundados sobre $\mathrm{o}$ assunto.

Dessa maneira, sugere-se a realização de estudos direcionados à prática clínica, além de estudos de caso comparativos entre grupos de mulheres sujeitas ao tratamento único da DMRA com Pilates em contrapartida a um grupo que utilize unicamente a fisioterapia convencional, para obtenção de resultados autênticos, fazendo com que tal assunto receba maior credibilidade e alcance um número significativo de mulheres.

\section{Referências}

Bom-fim, F. C., Martins, T. \& Fernandes, I. (2020). Efeito do método Pilates no tratamento de mulheres com diástase do músculo reto abdominal. Biblioteca Digital de TCC - UniAmérica, 1-24. https://pleiade.uniamerica.br/index.php/bibliotecadigital/article/view/577/676.

Bráz, Y. L. O. S., Silva, S. B. \& Silva, G. C. L. (2018). Recursos Fisioterapêuticos na Reabilitação da diástase no puerpério: uma revisão sistemática. In: III CONBRACIS será realizado em Campina Grande http://www.editorarealize.com.br/editora/anais/conbracis/2018/ TRABALHO_EV108_MD1_SA9_ID55_20052018160923.pdf.

Cursos Tao Pilates Joinville. O que é Diástase Abdominal? http://cursostaopilatesjoinville.blogspot.com/2016/06/o-que-e-diastase-abdominal.html.

Fei, H., Liu, Y., Li, M., He, J., Liu, L., Li, J., Wan, Y. \& Li, T. (2021). The relationship of severity in diastasis recti abdominis and pelvic floor dysfunction: a retrospective cohort study. BMC Women's Health, 21(1). https://www.ncbi.nlm.nih.gov/pmc/articles/PMC7885475/.

Fernandes, K. T. M. S. \& Santos, R. N. (2016). Os benefícios do método pilates no fortalecimento do assoalho pélvico no período gestacional: uma revisão bibliográfica. Rev Cien Escol Estad Saud Publ Cândido Santiago-RESAP, 2(3), 152-162. https://www.revista.esap.go.gov.b r/index.php/resap/article/view/38/53.

FisioStudio Pilates \& Fisioterapia. Diástase do abdome: tudo o que você precisa saber. https://fisiostudiopilates.com.br/diastase-do-abdome-tudo-o-que-voceprecisa-saber/.

HSN blog. Diástase Abdominal: O que é, como prevenir e falsos mitos. https://www.hsnstore.pt/blog/saude-e-beleza/lesoes/diastase-abdominal/.

Jessen, M. L., Öberg, S. \& Rosenberg, J. (2019). Treatment Options for Abdominal Rectus Diastasis. Frontiers in Surgery, 6, 65. https://pubmed.ncbi.nlm.nih.gov/31803753/.

Kroetz, D. C. \& Santos, M. D. (2015). Benefícios do método Pilates nas alterações musculoesqueléticas decorrentes do período gestacional. Revista Visão Universitária, 3(1). http://www.visaouniversitaria.com.br/ojs/index.php/home/article/view/62. 
Leite, A. C. N. M. T. \& Araújo, K. K. B. C. (2012). Diástase dos retos abdominais em puérperas e sua relação com variáveis obstétricas. Fisioterapia em Movimento, 25(2), 389-97. https://www.scielo.br/scielo.php?pid=S0103-51502012000200017\&script=sci_arttext\&tlng=pt.

Luna, D. C. B., Cavalcanti, A. L. A. M. H., Guendler, J. A, Brito, V. C. \& Oliveira, B. D. R. (2012). Frequência da diástase abdominal em puérperas e fatores de risco associados. Rev. Fisioter. S. Fun, 1(2), 10-17. http://www.repositorio.ufc.br/handle/riufc/13384.

Lynders, C. (2019). The Critical Role of Development of the Transversus Abdominis in the Prevention and Treatment of Low Back Pain. HSS Journal: the musculoskeletal journal of Hospital for Special Surgery, 15(3), 214-220. https://www.ncbi.nlm.nih.gov/pmc/articles/PMC6778169/

Macedo, A. G. P., Oliveira, C. L., Gonçalves, G. I. F. \& Mascarenhas, C. M. (2018). O Pilates e a gestação: uma relação de bem-estar. Revista de Trabalhos Acadêmicos - Campus Niterói, $\quad \mathrm{n}^{\circ} \quad 15 . \quad \mathrm{http}: / / \mathrm{www}$. revista.universo.edu.br/index.php?journal=1reta2\&page=article\&o $\quad \mathrm{p}=\mathrm{view} \& \mathrm{path} \% 5$ $\mathrm{B} \% 5 \mathrm{D}=6668 \&$ path $\% 5 \mathrm{~B} \% 5 \mathrm{D}=3398$.

Mommers, E. H. H., Ponten, J. E. H., Al Omar, A. K., Vries Reilingh, T. S., Bouvy, N. D. \& Nienhuijs, S. W. (2017). The general surgeon's perspective of rectus diastasis. A systematic review of treatment options. Surgical Endoscopy, 31(12), 4934-4949. https://www.ncbi.nlm.nih.gov/pmc/articles/PMC5715079/

Müller, I. A. S. \& Silva, K. V. M. (2018). Intervenção fisioterapêutica e medida dinâmica na diástase do reto abdominal: uma revisão sistemática. Biblioteca Universitária da UFJF. https://www.ufjf.br/facfisio/files/2019/09/TCC-Isabelle-M\%C3\%BCller-e-Karina-Silva.pdf.

Nahabedian, M. Y. (2018). Management Strategies for Diastasis Recti. Seminars in Plastic Surgery, 32(03), 147-154. https://pubmed.ncbi.nlm.nih.gov/30046291/

Nascimento, T. G. \& Mejia, D. P. M. (n.d.). O método pilates aplicado à ginecologia obstetrícia: benefícios durante o período gestacional e para o parto natural. Portal BioCursos. https://portalbiocursos.com.br/ohs/data/docs/35/17_-_O_mYtodo_pilates_aplicado_Y_ginecologia_obstetrYcia.pdf.

Pereira, A. S., Shitsuka, D. M., Parreira, F. J. \& Shitsuka, R. (2018). Metodologia da pesquisa científica. Repositório Digital da UFSM. https://repositorio.ufsm.br/handle/1/15824.

Pereira, N. S., Rego, B. A. C., Pinto, L. F. R. \& Blanco, K. B. (2020). Os benefícios do método Pilates diante das alterações do período gestacional. Revista Cathedral, 2(4), 50-60. http://cathedral.ojs.galoa.com.br/index.php/cathedral/article/view/223.

Pilates Center. O método Pilates. https://www.pilatescenterbh.com.br/pilates/.

PróFono. Paquímetro Digital Pró-Fono. https://profono.com.br/loja/paquimetro-digital-pro-fono/.

Silva, P. S. P., Filho, Campelo, S. R. S., Silva, O. F., Filho, Costa, J. V. S., Carvalho, A., Silva, W. G. \& et al. (2019). Efeito do método Pilates durante período gestacional. Atenção Interdisciplinar em Saúde, (Vol.1, pp. 229-236). Atena Editora. https://www.atenaeditora.com.br/post-artigo/25719.

Urbano, F. A., Barbosa, J. E. R., Junior, V. D. F., Martelli, A. \& Delbim, L. (2019). Exercícios de fortalecimento para o músculo reto abdominal como tratamento da diástase pós-gestacional. Revista Ciência e Saúde On-line, 4(1), 10-16. https://revistaeletronicafunvic.org/index.php/c14ffd10/article/view/117.

Vasconcelos, E. H., Oliveira, I. R. S., Alves, I. K., Santos, L. K. P., Silva, T. E. \& Lira, J. J. (2017). A intervenção fisiorepêutica na diástase do musculo reto abdominal (DMRA). Rev, Saberes, 6, 1. https://facsaopaulo.edu.br/wp-content/uploads/sites/16/2018/05/ed6especial/3.pdf. 